\title{
Transport in the static diffusion cloud chamber revisited
}

M. P. Anisimov

S. D. Shandakov

Y. I. Polygalov

Richard H. Heist

Fairfield University, rheist@fairfield.edu

Follow this and additional works at: https://digitalcommons.fairfield.edu/engineering-facultypubs Copyright 2001 American Institute of Physics

The final publisher PDF has been archived here with permission from the copyright holder.

Peer Reviewed

\section{Repository Citation}

Anisimov, M. P.; Shandakov, S. D.; Polygalov, Y. I.; and Heist, Richard H., "Transport in the static diffusion cloud chamber revisited" (2001). Engineering Faculty Publications. 141.

https://digitalcommons.fairfield.edu/engineering-facultypubs/141

\section{Published Citation}

Anisimov, M. P., Shandakov, S. D., Polygalov, Y. I., \& Heist, R. H. (2001). Transport in the static diffusion cloud chamber revisited. The Journal of Chemical Physics, 114(2), 899-906. doi:10.1063/1.1318735.

This item has been accepted for inclusion in DigitalCommons@Fairfield by an authorized administrator of DigitalCommons@Fairfield. It is brought to you by DigitalCommons@Fairfield with permission from the rightsholder(s) and is protected by copyright and/or related rights. You are free to use this item in any way that is permitted by the copyright and related rights legislation that applies to your use. For other uses, you need to obtain permission from the rights-holder(s) directly, unless additional rights are indicated by a Creative Commons license in the record and/or on the work itself. For more information, please contact digitalcommons@fairfield.edu. 


\title{
Transport in the static diffusion cloud chamber revisited
}

\author{
M. P. Anisimov \\ Aerosol Nucleation Laboratory at Kemerovo, Institute of Catalysis, SB RAS, Russia \\ and Department of Chemical Engineering, Clarkson University, Potsdam, New York 13699-5810 \\ S. D. Shandakov and Yu. I. Polygalov
Kemerovo State University, 6 Krasnaya Str., 650043 Kemerovo, Russia
R. H. Heist
Nucleation Laboratory, Manhattan College, Riverdale, New York 10471-4098
}

(Received 24 July 2000; accepted 25 August 2000)

\begin{abstract}
The static diffusion chamber (SDC) allows the measurement of critical supersaturation and of nucleation rates and it is a powerful instrument for the vapor nucleation study. Earlier, within the scope of the International Nucleation Workshop Group, nucleation rates of the $n$-pentanol-helium system have been measured using different experimental techniques. Disagreement of experimental data obtained using the static diffusion chamber and data obtained using other methods, particularly the laminar flow diffusion chamber, can be explained by re-examining the mass and energy transport analysis used to describe static diffusion chamber operation. In the present research we describe the mass and energy transport in the SDC modeled as an effectively open system with mass and energy transport in one direction with a nonzero diffusion flux at the system boundaries. Calculated values for vapor supersaturation are compared with the $n$-pentanol nucleation rate experimental results of the American-Czech group [M. Rudek, J. L. Katz, I. Y. Vidensky et al., J. Chem. Phys. 111, 3623 (1999)] and with a nucleation rate Reference Equation obtained from an earlier investigation involving the $n$-pentanol-helium system. From our results one can see that there is a significant difference in the calculated supersaturation for all of the data. The magnitude of this difference is quite large even for the relatively small vapor mass fractions at a nucleation temperature of $260 \mathrm{~K}$. We also note that the calculated nucleation temperatures from our analysis are slightly larger than those reported in the work of Rudek et al. ${ }^{4} \mathrm{We}$ performed our calculations with and without the thermal diffusion term. We observed that the effect of thermal diffusion on the transport process is relativelly small and is not particularly essential to include in this comparison that we are making the effects of the different flux boundary conditions. (C) 2001 American Institute of Physics. [DOI: 10.1063/1.1318735]
\end{abstract}

\section{INTRODUCTION}

Experimental research of homogeneous nucleation kinetics in supersaturated vapors is based on the determination of the relation between nucleation rate and, as a rule, vapor supersaturation (activity) while maintaining other parameters constant. The static diffusion chamber (SDC) allows measurement of critical supersaturation and of nucleation rates and it is a powerful instrument for the vapor nucleation study. Within the scope of the International Nucleation Workshop Group, ${ }^{1}$ nucleation rates of the $n$-pentanolhelium system have been measured using different experimental techniques. ${ }^{2-5}$ Disagreement of experimental data obtained using the static diffusion chamber and data obtained using other methods, particularly the laminar flow diffusion chamber, can be explained by re-examining the mass and energy transport analysis used to describe static diffusion chamber operation. There appear to be no other reasons for this disagreement, since the sample working fluid and the system parameters were the same for all groups. Recently the effect of the radial vapor flow to chamber sidewall ${ }^{6,7}$ has been discussed. Good agreement between one-dimensional and two-dimensional models (with zero mass average velocities at boundary) has been achieved.
It is customary to consider that the diffusion flux is zero at the chamber boundaries where the vapor is in equilibrium with the wet surfaces. However, the SDC can be considered as an open system along the direction of transport of vapor from the hot surface boundary to the cold surface boundary. In this model, the assumption of a zero vapor flux to a boundary surface is not correct. In this case, zero vapor flux may be only be realized in the case of zero vapor concentration. For the case of nonzero vapor concentration there are three boundary conditions that are required for solution of the second order differential equation with two integration constants, i.e., the first two are the equilibrium vapor concentrations at the hot and cold surfaces and the third is the zero value for the vapor diffusion flux. Recently the nonzero diffusion vapor flux to the boundary surfaces was accounted for in the initial transport equations. ${ }^{8}$ The resulting numerical solution $^{8}$ is in a good agreement with the existing onedimensional model based on a numerical solution of the Stefan-Maxwell and heat transfer equations.

In the research reported here we describe the mass and energy transport in the SDC modeled as an open system with mass and energy transport in one direction with a nonzero diffusion flux at the system boundaries. Calculated values for 
vapor supersaturation are compared with the $n$-pentanol nucleation rate experimental results of the American-Czech group $^{4}$ and with a nucleation rate Reference Equation obtained from an earlier investigation involving the $n$-pentanol-helium system. ${ }^{5}$

\section{SYSTEM EQUATIONS}

To describe the heat and mass transfer in the SDC (or the flow diffusion chamber), we apply the Navier-Stokes equations for a system with axial symmetry and stationary flow. The heat and mass transfer equations in vector form may be found in the monographs ${ }^{9,10}$ listed in the References. A specific application of these equations for the system under consideration here is presented in the Appendix. Using differential operators expressed in the cylindrical coordinate system, the heat and mass transport equations may be presented as

$$
\begin{aligned}
\frac{\partial}{\partial z}(\rho u)+\frac{1}{r} \frac{\partial}{\partial r}(r \rho v)=0 & \\
\rho u \frac{\partial c}{\partial z}+\rho v \frac{\partial c}{\partial r}= & -\frac{1}{r} \frac{\partial r J_{r 2}}{\partial r}-\frac{\partial J_{z 2}}{\partial z} \\
\rho u \frac{\partial u}{\partial z}+\rho v \frac{\partial u}{\partial r}= & -\frac{\partial p}{\partial z}+\frac{1}{r} \frac{\partial}{\partial r}\left(r \mu \frac{\partial u}{\partial r}\right) \\
& +\frac{4}{3} \frac{\partial}{\partial z}\left(\mu \frac{\partial u}{\partial z}\right)+\frac{1}{r} \frac{\partial}{\partial r}\left(r \mu \frac{\partial v}{\partial z}\right) \\
& -\frac{2}{3} \cdot \frac{1}{r} \frac{\partial}{\partial z}\left(\mu \frac{\partial(r v)}{\partial r}\right)+\rho g \\
\rho u \frac{\partial v}{\partial z}+\rho v \frac{\partial v}{\partial r}= & -\frac{\partial p}{\partial r}+\frac{\partial}{\partial z}\left(\mu \frac{\partial u}{\partial r}\right) \\
& +\frac{4}{3 r} \frac{\partial}{\partial r}\left(r \mu \frac{\partial v}{\partial r}\right)+\frac{\partial}{\partial z}\left(\mu \frac{\partial v}{\partial z}\right) \\
& -\frac{2}{3}\left[\frac{\partial}{\partial r}\left(\mu \frac{\partial u}{\partial z}\right)+\frac{v}{r}\left(\frac{\partial \mu}{\partial r}-\frac{\mu}{r}\right)\right] \\
& \left.+\left(\frac{\partial u}{\partial z}\right)^{2}-\frac{1}{3}\left(\frac{v}{r}+\frac{\partial v}{\partial r}+\frac{\partial u}{\partial z}\right)^{2}\right] \\
& -\frac{\partial}{\partial z} q z+2 \mu\left(\frac{1}{\partial z}\left(\frac{\partial u}{\partial r}+\frac{\partial v}{\partial z}\right)^{2}+\left(\frac{\partial v}{\partial r}\right)^{2}\right. \\
\rho u \frac{\partial}{\partial z}+\rho v \frac{\partial}{\partial r}(r q) &
\end{aligned}
$$

Here Eqs. (1) and (2) are continuity equations for the mixture and the vapor, respectively; Eqs. (3) and (4) are the equations of motion in the axial and radial directions, respectively; Eq. (5) is the energy equation. The expressions for the vapor mass diffusion flux and heat flux in the radial and axial directions, respectively, can be written in the form,

$$
\begin{aligned}
J_{r 2}= & -c \rho D_{12}\left[\left(\frac{\partial \ln c}{\partial r}+(1-c) \frac{M_{1}-M_{2}}{M} \frac{\partial \ln p}{\partial r}\right)\right. \\
& \left.+\frac{k_{T 2}}{c} \frac{M_{1} M_{2}}{M^{2}} \frac{\partial \ln T}{\partial r}\right], \\
J_{z 2}= & -c \rho D_{12}\left[\left(\frac{\partial \ln c}{\partial z}+(1-c) \frac{M_{1}-M_{2}}{M} \frac{\partial \ln p}{\partial z}\right)\right. \\
& \left.+\frac{k_{T 2}}{c} \frac{M_{1} M_{2}}{M^{2}} \frac{\partial \ln T}{\partial z}\right], \\
q_{r}= & -\lambda \frac{\partial T}{\partial r}+\left(h_{2}-h_{1}+\frac{k_{T 2} R_{g} T}{c(1-c) M}\right) \cdot J_{r 2}, \\
q_{z}= & -\lambda \frac{\partial T}{\partial z}+\left(h_{2}-h_{1}+\frac{k_{T 2} R_{g} T}{c(1-c) M}\right) \cdot J_{z 2} .
\end{aligned}
$$

The above system of equations can be applied to the description of any cylindrically symmetric heat and mass transfer process. The specific features of such process are made apparent through the proper choice of boundary conditions. In our present discussion a heat and mass transfer problem in the SDC is considered. To complete the system of Eqs. (1)(9) the equation of state for an ideal gas is used in the form,

$$
p=\rho R_{g} T / M .
$$

Here $R_{g}$ is gas universal constant. Other nomenclature, used in this work is presented in Table I.

\section{BOUNDARY CONDITIONS}

The static diffusion chamber consists of two wet surfaces, separated by ring with radius, $R$, and height, $H$. It is assumed that at the hot surface with temperature, $T_{0}$, and at the cold surface with temperature, $T_{1}$, the vapor concentrations are equal to the saturated (equilibrium) vapor concentrations, $c_{0}$ and $c_{1}$, respectively,

$$
(c)_{z=0}=c_{\mathrm{sat}}\left(T_{0}\right)=c_{0}, \quad(c)_{z=H}=c_{\mathrm{sat}}\left(T_{1}\right)=c_{1} .
$$

The mass average velocity at either surface is determined from condition that the background gas does not penetrate either wet surface. At $z=0$ this condition gives

$$
\left(1-c_{0}\right) \rho_{0} u_{0}-J_{z 0}=0 .
$$

As it is mentioned above, the SDC can be considered as an (effectively) open system in one direction because the mass flux of vapor passes from one boundary (hot plate) to the other boundary (cold plate). For this reason the concept of using a zero mass average velocity for the vapor flux at either system boundary is not desirable, so in the present work we employ the conditions shown in Eq. (11) along with a nonzero diffusion flux of the vapor through the system boundaries, i.e., Eq. (12). To ensure a negligible radial vapor diffusion flux component relative to the axial component we employ a large diameter to height ratio, e.g., $D / H=10$, see 
TABLE I. Nomenclature and the reduced variables.

\begin{tabular}{|c|c|c|}
\hline Nomenclature & Symbol & Reduced variables \\
\hline $\begin{array}{l}\text { Vapor mass } \\
\text { fraction }\end{array}$ & $c$ & \\
\hline $\begin{array}{l}\text { Coordinate in } \\
\text { axial direction } \\
\text { from bottom }\end{array}$ & $z$ & $H$ \\
\hline $\begin{array}{l}\text { Coordinate in } \\
\text { radial direction } \\
\text { from axis }\end{array}$ & $r$ & $R$ \\
\hline $\begin{array}{l}\text { Axial mass } \\
\text { diffusion flux } \\
\text { of vapor }\end{array}$ & $J_{z 2}$ & $\begin{array}{l}J_{z 2}^{0}=\bar{J}_{20} \rho_{0} D_{12}^{0} / H \\
\tilde{j}_{z 0}=(\partial c / \partial \bar{z})_{0}+\bar{M}_{1} \bar{M}_{2} k_{T 2}^{0}(\partial \bar{T} / \partial \bar{z})_{0}\end{array}$ \\
\hline $\begin{array}{l}\text { Radial mass } \\
\text { diffusion flux } \\
\text { of vapor }\end{array}$ & $J_{r 2}$ & $J_{r 2}^{0}=J_{z 2}^{0}(H / R)$ \\
\hline Axial heat flux & $q_{z}$ & $\begin{array}{l}q_{z 0}=\bar{q}_{z 0} \lambda_{0} T_{0} / H \\
\bar{q}_{z 0}=-\left(\frac{\partial \bar{T}}{\partial \bar{z}}\right)_{0}+\operatorname{Le}_{0}^{-1}\left(\bar{h}_{2}-\bar{h}_{1}+\frac{k_{T 2}^{0}}{c_{0}\left(1-c_{0}\right)} \frac{R_{g}}{M_{0} c_{P 0}}\right) \bar{j}_{z 0}\end{array}$ \\
\hline Radial heat flux & $q_{r}$ & $q_{r o}=q_{z}^{0}(H / R)$ \\
\hline Axial velocity & $u$ & $u_{0}=J_{z 2}^{0} / \rho_{0}\left(1-c_{0}\right)$ \\
\hline Radial velocity & $v$ & $v_{0}=u_{0}(H / R)$ \\
\hline Total pressure & $P$ & $P_{0}$ \\
\hline Temperature & $T$ & $T_{0}$ \\
\hline $\begin{array}{l}\text { Density of } \\
\text { mixture }\end{array}$ & $\rho$ & $\rho_{0}=M_{0} P_{0} /\left(R_{g} T_{0}\right)$ \\
\hline $\begin{array}{l}\text { Molar mass of } \\
\text { gas, vapor, and } \\
\text { mixture }\end{array}$ & $M_{1}, M_{2}, M$ & $M_{0}=\left(\frac{1-c_{0}}{M_{1}}+\frac{c_{0}}{M_{2}}\right)^{-1}$ \\
\hline $\begin{array}{l}\text { Viscosity of gas, } \\
\text { vapor, mixture }\end{array}$ & $\mu_{1}, \mu_{2}, \mu$ & $\mu_{0}=\mu\left(c_{0}, T_{0}\right)$ \\
\hline $\begin{array}{l}\text { Binary diffusion } \\
\text { coefficient }\end{array}$ & $D_{12}$ & $D_{12}^{0}=D_{12}\left(c_{0}, T_{0}\right)$ \\
\hline $\begin{array}{l}\text { Thermodiffusion } \\
\text { factor for vapor }\end{array}$ & $k_{T 2}$ or $\alpha_{12}$ & $k_{T 2}^{0}=k_{T 2}\left(c_{0}, T_{0}\right)$ or $\alpha_{12}^{0}=\alpha_{12}\left(c_{0}, T_{0}\right)$ \\
\hline $\begin{array}{l}\text { Thermal } \\
\text { conductivity of } \\
\text { gas, vapor, } \\
\text { mixture }\end{array}$ & $\lambda_{1}, \lambda_{2}, \lambda$ & $\lambda_{0}=\lambda\left(c_{0}, T_{0}\right)$ \\
\hline $\begin{array}{l}\text { Specific heat } \\
\text { capacity at } \\
\text { constant pressure }\end{array}$ & $c_{P 1}, c_{P 2}, c_{p}$ & $c_{P 0}=\left(1-c_{0}\right) c_{P 1}^{0}+c_{0} c_{P 2}^{0}$ \\
\hline $\begin{array}{l}\text { Specific enthalpy } \\
\text { of gas, vapor, } \\
\text { mixture }\end{array}$ & $h_{1}, h_{2}, h$ & $h_{0}=c_{P 0} T_{0}$ \\
\hline Collision integral & $\Omega^{(i, j)}\left(k T / \varepsilon_{i j}\right)$ & $\Omega^{(i, j)}\left(k T_{0} / \varepsilon_{i j}\right)$ \\
\hline
\end{tabular}

Ref. 6(a) for details. This conditions also permits the use of a one-dimensional approximation for the heat and mass transport processes in the SDC.

To simplify the initial governing equations, it is convenient to reduce the equations to dimensionless form using the driving forces and the chamber size as the scaling factors.

\section{DIMENSIONLESS FORM OF THE SYSTEM EQUATIONS}

According to the flux boundary conditions, the axial vapor and heat fluxes in the SDC appear as the gradients of vapor concentration and temperature between the surfaces. Thus, the driving forces in the axial direction are due to these gradients. Hence scaling factors for the axial vapor diffusion and heat fluxes (as primary values) may be defined from Eqs. (7) and (9), respectively, as

$$
\begin{aligned}
J_{z 0}= & \left(J_{z 2}\right)_{z=0}=\bar{j}_{z 0} \rho_{0} D_{12}^{0} / H \\
\bar{j}_{z 0}= & (\partial c / \partial \bar{z})_{0}+\bar{M}_{1} \bar{M}_{2} k_{T 2}^{0}(\partial \bar{T} / \partial \bar{z})_{0}+c_{0}\left(1-c_{0}\right)\left(\bar{M}_{1}\right. \\
& \left.-\bar{M}_{2}\right)(\partial \bar{p} / \partial \bar{z})_{0} \\
q_{z 0}= & \left(q_{z 0}\right)_{z=0}=\bar{q}_{z 0} \lambda_{0} T_{0} / H \\
\bar{q}_{z 0}= & -(\partial \bar{T} / \partial \bar{z})_{0}+L e_{0}^{-1} \\
& \times\left(\bar{h}_{20}-\bar{h}_{10}+\frac{k_{T 2}^{0}}{c_{0}\left(1-c_{0}\right)} \frac{R_{g}}{M_{0} c_{P 0}}\right) \bar{j}_{z 0} .
\end{aligned}
$$

Assuming that the appropriate gradients in the radial directions are of the same order as in the axial directions, we obtain the analogous expressions, (7) and (8), for the radial vapor diffusion and heat fluxes. Here we use the height, $H$, 
and radius, $R$, of the SDC as length scales in the axial and radial directions, respectively. The scale of axial velocity (as a secondary value) is defined according to the boundary condition (12). The definitions of all the scaling factors used in this analysis are shown in Table I. Using these scaling factors we rewrite the system equations (1)-(5) in dimensionless form as

$$
\begin{aligned}
& \frac{\partial}{\partial \bar{z}}(\bar{\rho} \bar{u})+\varepsilon^{2} \frac{1}{\bar{r}} \frac{\partial}{\partial \bar{r}}(\bar{r} \bar{\imath} \bar{v})=0, \\
& \bar{\rho} \bar{u} \frac{\partial c}{\partial \bar{z}}+\varepsilon^{2} \bar{\rho} \bar{v} \frac{\partial c}{\partial \bar{r}}=-\left(1-c_{0}\right)\left(\frac{\partial \bar{J}_{z 2}}{\partial \bar{z}}+\varepsilon^{2} \frac{1}{\bar{r}} \frac{\partial \bar{r} \bar{J}_{r 2}}{\partial \bar{r}}\right), \\
& \bar{\rho} \bar{u} \frac{\partial \bar{u}}{\partial \bar{z}}+\varepsilon^{2} \bar{\rho} \bar{v} \frac{\partial \bar{u}}{\partial \bar{r}}=-\mathrm{Eu} \frac{\partial \bar{p}}{\partial \bar{z}}+\frac{1}{\mathrm{Fr}} \bar{\rho} \bar{g}+\mathrm{Sc} \frac{1-c_{0}}{c_{0}} \\
& \times\left(\frac{4}{3} \frac{\partial}{\partial \bar{z}}\left(\bar{\mu} \frac{\partial \bar{u}}{\partial \bar{z}}\right)+\varepsilon^{2} \frac{1}{\bar{r}} \frac{\partial}{\partial \bar{r}}\left(\bar{r} \bar{\mu} \frac{\partial \bar{u}}{\partial \bar{r}}\right)\right. \\
& +\varepsilon^{2} \frac{1}{\bar{r}} \frac{\partial}{\partial \bar{r}}\left(\bar{r} \bar{\mu} \frac{\partial \bar{v}}{\partial \bar{z}}\right) \\
& \left.-\varepsilon^{2} \frac{2}{3} \frac{1}{\bar{r}} \frac{\partial}{\partial \bar{z}}\left(\bar{\mu} \frac{\partial(\bar{r} \bar{v})}{\partial \bar{r}}\right)\right) \text {, } \\
& \bar{\rho} \bar{u} \frac{\partial \bar{v}}{\partial \bar{z}}+\varepsilon^{2} \bar{\rho} \bar{v} \frac{\partial \bar{v}}{\partial \bar{r}}=-\operatorname{Er} \frac{\partial \bar{p}}{\partial \bar{r}}+\operatorname{Sc} \frac{1-c_{0}}{c_{0}}\left(\frac{\partial}{\partial \bar{z}}\left(\bar{\mu} \frac{\partial \bar{u}}{\partial \bar{r}}\right)\right. \\
& +\frac{\partial}{\partial \bar{z}}\left(\bar{\mu} \frac{\partial \bar{v}}{\partial \bar{z}}\right)-\frac{2}{3} \frac{\partial}{\partial \bar{r}}\left(\bar{\mu} \frac{\partial \bar{u}}{\partial \bar{z}}\right) \\
& +\varepsilon^{2} \frac{4}{3 \bar{r}} \frac{\partial}{\partial \bar{r}}\left(\bar{r} \bar{\mu} \frac{\partial \bar{v}}{\partial \bar{r}}\right) \\
& \left.-\varepsilon^{2} \frac{2}{3} \frac{\bar{v}}{\bar{r}}\left(\frac{\partial \bar{\mu}}{\partial \bar{r}}-\frac{\bar{\mu}}{\bar{r}}\right)\right) \\
& \bar{\rho} \bar{u} \frac{\partial \bar{h}}{\partial \bar{z}}+\varepsilon^{2} \bar{\rho} \bar{v} \frac{\partial \bar{h}}{\partial \bar{r}}=-\mathrm{Le}_{0}\left(1-c_{0}\right) \frac{\bar{q}_{z 0}}{\bar{j}_{z 0}}\left(\frac{\partial \bar{q}_{z}}{\partial \bar{z}}+\varepsilon^{2} \frac{1}{\bar{r}} \frac{\partial\left(\bar{r} \bar{q}_{r}\right)}{\partial \bar{r}}\right) \\
& +\frac{R_{g}}{M_{0} c_{P 0}}\left(\bar{u} \frac{\partial \bar{p}}{\partial \bar{z}}+\varepsilon^{2} \bar{v} \frac{\partial \bar{p}}{\partial \bar{r}}\right) \\
& +\frac{2 \bar{\mu}}{\operatorname{Re} \operatorname{Eu}} \frac{R_{g}}{M_{0} c_{P 0}}\left[\left(\frac{\partial \bar{u}}{\partial \bar{z}}\right)^{2}\right. \\
& +\varepsilon^{2} \frac{1}{2}\left(\frac{\partial \bar{u}}{\partial \bar{r}}+\frac{\partial \bar{v}}{\partial \bar{z}}\right)^{2}+\varepsilon^{4}\left(\frac{\partial \bar{v}}{\partial \bar{r}}\right)^{2} \\
& \left.-\frac{1}{3}\left(\varepsilon^{2} \frac{\bar{v}}{\bar{r}}+\varepsilon^{2} \frac{\partial \bar{v}}{\partial \bar{r}}+\frac{\partial \bar{u}}{\partial \bar{z}}\right)^{2}\right] \text {. }
\end{aligned}
$$

$$
\begin{aligned}
\frac{\partial \bar{p}}{\partial \bar{z}} & =0, \quad \frac{\partial \bar{p}}{\partial \bar{r}}=0, \quad \bar{\rho} \bar{T} / \bar{M}=1, \\
\bar{\rho} \bar{u} \frac{\partial \bar{h}}{\partial \bar{z}}+\varepsilon^{2} \bar{\rho} \bar{v} \frac{\partial \bar{h}}{\partial \bar{r}} & \\
& =-L e_{0}\left(1-c_{0}\right) \frac{\bar{q}_{z 0}}{\bar{j}_{z 0}}\left(\frac{\partial}{\partial \bar{z}} \bar{q}_{z}+\varepsilon^{2} \frac{1}{\bar{r}} \frac{\partial}{\partial \bar{r}}\left(\bar{r} \bar{q}_{r}\right)\right) .
\end{aligned}
$$

The equations of motion may be presented in the form (18) because the pressure drop is taken to be zero at very large Euler number. It should be noted, however, that in the flow diffusion chamber even though the axial pressure gradient is small it cannot be neglected since in that case it is a driving force.

The system equations, (15)-(19), with appropriate boundary conditions, can be solved numerically. Boundary conditions appropriate for the experimental system under study are given in dimensionless form as

(1) At bottom (hot) plate,

$$
c=c_{0}, \bar{T}=1, \bar{u}=1, \bar{v}=0 \text { at } \bar{z}=0 ;
$$

(2) At top (cold) plate,

$$
c=c_{1}, \bar{T}=\tau \text { at } \bar{z}=1
$$

(3) At $\bar{r}=1$ :

wet wall: $c=c_{\mathrm{eq}}\left(T_{W}\right), \vec{T}=\bar{T}_{W}, \bar{u}=0, \bar{v}=\bar{J}_{r 2}\left(1-c_{0}\right) / \bar{\rho}(1$ $\left.-c_{\text {eq }}\left(T_{W}\right)\right), \bar{q}_{r}=0$; dry wall: $\bar{J}_{r 2}=0, \bar{T}=\bar{T}_{W}, \bar{u}=0, \bar{v}=0$, $\bar{q}_{r}=\bar{q}_{W}(\bar{z})$.

Here we have assumed that temperature dependence, $\bar{T}_{W}(\bar{z})$, and the additional heating flux, $\bar{q}_{W}(\bar{z})$ to keep the wall dry are known and that the presence of the liquid flow down the sidewall is neglected. However, we note that the liquid film motion along the wall may not be simple in the case of a wet wall when the vapor concentration gradient is not uniform in the presence of the sidewall. For the purpose of present work, namely, to show the effect of a nonzero axial vapor diffusion flux, we discuss below simplifications to the equations describing the heat and mass transport in the SDC.

If we consider to small order the size ratio $\left(\varepsilon^{2} \sim 10^{-1}\right.$, see Ref. 4), then the system of heat and mass transport equations may be written to an accuracy of $O\left(\varepsilon^{2}\right)$ as

$$
\frac{\partial}{\partial \bar{z}}(\bar{\rho} \bar{u})=0, \quad \bar{\rho} \bar{u} \frac{\partial c}{\partial \bar{z}}=-\left(1-c_{0}\right) \frac{\partial \bar{J}_{z 2}}{\partial \bar{z}},
$$




$$
\begin{aligned}
\bar{\rho} \bar{u} \frac{\partial \bar{h}}{\partial \bar{z}}= & -\operatorname{Le}_{0}\left(1-c_{0}\right) \frac{\bar{q}_{z 0}}{\bar{j}_{z 0}} \frac{\partial}{\partial \bar{z}} \bar{q}_{z} \\
\bar{J}_{z 2} \bar{j}_{z 0}= & -\bar{\rho} \bar{D}_{12}\left(\frac{\partial c}{\partial \bar{z}}+\frac{k_{T 2}}{\bar{T}} \frac{\bar{M}_{1} \bar{M}_{2}}{\bar{M}^{2}} \frac{\partial \bar{T}}{\partial \bar{z}}\right) \\
\bar{q}_{z} \bar{q}_{z 0}= & -\bar{\lambda} \frac{\partial \bar{T}}{\partial \bar{z}} \\
& +\mathrm{Le}_{0}^{-1}\left(\bar{h}_{2}-\bar{h}_{1}+\frac{k_{T 2}}{c(1-c)} \frac{\bar{T}}{\bar{M}} \frac{R_{g}}{M_{0} c_{P 0}}\right) \bar{J}_{z 2} \bar{j}_{z 0} .
\end{aligned}
$$

We show below that this system of equations with boundary conditions (20) and (21) admits to an analytical solution for the case of light carrier gas. tion,

We note that for the dry wall condition the energy equa-

$$
\bar{\rho} \bar{u} \frac{\partial \bar{h}}{\partial \bar{z}}=-\operatorname{Le}_{0}\left(1-c_{0}\right) \frac{\bar{q}_{z 0}}{\bar{j}_{z 0}} \frac{\partial}{\partial \bar{z}}\left(\bar{q}_{z}+\varepsilon^{2} \frac{1}{\bar{r}} \frac{\partial}{\partial \bar{r}}\left(\bar{r} \bar{q}_{r}\right)\right)
$$

can be reduced to one-dimension form when the additional wall heating is represented such that radial heat flux is proportional to radius, $\bar{q}_{r} \sim \bar{r}$. We note here the frequent observations in the literature that in many SDC investigations there is a significant dependence of nucleation rate on this wall heating value. See Ref. 6(b) for a more detailed discussion of this point.

\section{ONE-DIMENSION CONSIDERATION}

After integration using the boundary condition (20), Eqs. (22) and (23) can be expressed as

$$
\begin{aligned}
& \bar{\rho} \bar{u}=1, \quad \bar{J}_{z 2}=(1-c) /\left(1-c_{0}\right), \\
& \left(\bar{h}-\bar{h}\left(c_{0}, T_{0}\right)\right) \bar{J}_{z 2} \bar{j}_{z 0} \frac{1-c_{0}}{1-c}=-\mathrm{Le}_{0}\left(1-c_{0}\right)\left(\bar{q}_{z} \bar{q}_{z 0}-\bar{q}_{z 0}\right) .
\end{aligned}
$$

Substituting Eqs. (25) and (26) into Eq. (27) and using Eq. (14) along with an expression for the specific enthalpy in form $\bar{h}(c, T)=(1-c) \bar{h}_{1}(T)+c \bar{h}_{2}(T)$ one obtains first,

$$
\begin{aligned}
\mathrm{Le}_{0}\left(1-c_{0}\right)\left(\bar{\lambda} \frac{\partial \bar{T}}{\partial \bar{z}}-\left(\frac{\partial \bar{T}}{\partial \bar{z}}\right)_{0}\right) \\
=\left(\bar{h}_{2}-\bar{h}_{20}+\frac{R_{g}}{M_{0} c_{P 0}} \frac{k_{T 2}^{0}}{c_{0}}\left(\frac{c_{0}}{c} \frac{1-c_{0}}{1-c} \frac{k_{T 2} \bar{T}}{k_{T 2}^{0} \bar{M}}-1\right)\right) \bar{j}_{z 0} .
\end{aligned}
$$

And, substituting the expression for vapor enthalpy one can rewrite Eq. (28) as

$$
\bar{\lambda} \frac{\partial \bar{T}}{\partial \bar{z}}=-\frac{\bar{j}_{z 0}}{1-c_{0}}\left(A^{-1}+B(c, \bar{T}) / \mathrm{Le}_{0}\right),
$$

$$
\begin{aligned}
B(c, \bar{T})= & \int_{\bar{T}}^{1} \bar{c}_{P 2} d \bar{T}+\frac{R_{g}}{M_{0} c_{P 0}} \frac{k_{T 2}^{0}}{c_{0}} \\
& \times\left(1-\frac{c_{0}}{c} \frac{1-c_{0}}{1-c} \frac{k_{T 2}}{k_{T 2}^{0}} \frac{\bar{T}}{\bar{M}}\right) .
\end{aligned}
$$

Here we designate the constant of integration as

$$
A^{-1}=-(\partial \bar{T} / \partial \bar{z})_{0}\left(1-c_{0}\right) / \bar{j}_{z 0} .
$$

Using Eqs. (24) and (26) one can express $\bar{j}_{z 0}$ as

$$
\bar{j}_{z 0}=-\bar{\rho} \bar{D}_{12} \frac{1-c_{0}}{1-c} \frac{\partial \bar{T}}{\partial \bar{z}}\left(\frac{\partial c}{\partial \bar{T}}+\frac{k_{T 2}}{\bar{T}} \frac{\bar{M}_{1} \bar{M}_{2}}{\bar{M}^{2}}\right) .
$$

Here it is assumed that $\partial \bar{T} / \partial \bar{z} \neq 0$. Substituting Eq. (30) into Eq. (29) and after a simple transformation we obtain

$$
\frac{d c}{d \bar{T}}=\frac{\bar{\lambda}}{\bar{\rho} \bar{D}_{12}}-\frac{A(1-c)}{1+\mathrm{Le}_{0}^{-1} A \cdot B(c, \bar{T})}-\frac{k_{T 2}}{\bar{T}} \frac{\bar{M}_{1} \bar{M}_{2}}{\bar{M}^{2}} .
$$

Thus, we have the first order differential equation, where the integration constant $A$ is defined using one of two boundary conditions for the vapor concentration at the surfaces with different temperatures, $T_{0}$ and $T_{1}$. Expressions for the mass and heat transfer coefficients, as well as other thermodynamic and hydrodynamic data used in Eq. (31) can be found in Hirschfelder et al. ${ }^{9}$ or Reid et al ${ }^{11}$ and the expressions used in this analysis are given in Table II. (In Table II, $N_{A}$ is the Avogadro number.) We note that the thermal diffusion factor for the vapor given by Hirschfelder et al. ${ }^{9} k_{T 2}$, and that by Rudek et al. ${ }^{4} \alpha_{12}$, are not the same and are related as

$$
k_{T 2} \frac{M_{1} M_{2}}{M^{2}}=\alpha_{12} c(1-c) .
$$

The approximation for $\alpha_{12}$ for the $n$-pentanol-helium system used in the present investigation is given in Table II. Equation (31), when expressed using factor $\alpha_{12}$, has the following form:

$$
\begin{aligned}
& \frac{d}{d \bar{T}}\left(\frac{1-c_{0}}{1-c}\right)=\frac{A Q(c, \bar{T})}{1+\mathrm{Le}_{0}^{-1} A \cdot B(c, \bar{T})}-c\left(\frac{1-c_{0}}{1-c}\right) \frac{\alpha_{12}}{\bar{T}}, \\
& B(c, \bar{T})=\int_{\bar{T}}^{1} \bar{c}_{P 2} d \bar{T}+\frac{R_{g}}{M_{0} c_{P 0}} \frac{1-c_{0}}{\bar{M}_{1} \bar{M}_{2}}\left(\alpha_{12}^{0}-\alpha_{12} \bar{T} \bar{M}\right), \\
& Q(c, \bar{T})=\frac{\bar{\lambda}}{\bar{\rho}_{12}} \frac{\left(1-c_{0}\right)}{(1-c)} .
\end{aligned}
$$

Here we have introduced the parameter, defined in Eq. (35) because when using the approximation of a light background gas $\left(M_{1} \ll M_{2}\right)$, it is only a slight function of temperature. Thus, we have $\lambda \rightarrow \lambda_{1}, M \rightarrow M_{1} /(1-c)$ (see Table II),

$$
\begin{aligned}
Q(c, T) & \rightarrow \frac{\Omega^{(2,2)}\left(k_{B} T / \varepsilon_{1}\right)}{\Omega^{(2,2)}\left(k_{B} T_{0} / \varepsilon_{1}\right)} \frac{\Omega^{(1,1)}\left(k_{B} T_{0} / \varepsilon_{12}\right)}{\Omega^{(2,2)}\left(k_{B} T / \varepsilon_{1}\right)} \\
& \approx 1 \text { at } M_{1} / M_{2} \rightarrow 0 .
\end{aligned}
$$


TABLE II. Thermophysical properties of helium, hydrogen, and $n$-pentanol and their mixtures.

\section{Helium}

$M_{1}=4.0026 \times 10^{-3} \mathrm{~kg} / \mathrm{mol}$

$\sigma_{1}=2.551 \times 10^{-10} \mathrm{~m}, \varepsilon_{11} / k_{B}=10.22 \mathrm{~K}$

$c_{P 1}=5 R_{g} / 2 M_{1} \mathrm{~J} \mathrm{~kg}^{-1} \mathrm{~K}^{-1}$

$\lambda_{1}=2.45108 \times 10^{-2}+1.1246 \times 10^{-3} \mathrm{~T}-2.93123 \times 10^{-6} \times T^{2}$

$+4.49646 \times 10^{-9} \times T^{3}-2.51948 \times 10^{-12} \times T^{4} \mathrm{~W} \mathrm{~m}^{-1} \mathrm{~K}^{-1}$

Hydrogen

$$
\begin{aligned}
M_{1}= & 2.016 \times 10^{-3} \mathrm{~kg} / \mathrm{mol} \\
\sigma_{1}= & 2.827 \times 10^{-10} \mathrm{~m}, \varepsilon_{11} / k_{B}=59.7 \mathrm{~K} \\
c_{P 1}= & 3.444 R_{g} / M_{1} \mathrm{~J} \mathrm{~kg}^{-1} \mathrm{~K}^{-1} \\
\lambda_{1}= & 418.68\left(5.468234 \times 10^{-5}+2.137513 \times 10^{-6}\right. \\
& \left.\times T-1.697643 \times 10^{-10} \times T^{2}\right) \mathrm{W} \mathrm{m}^{-1} \mathrm{~K}^{-1}
\end{aligned}
$$

$n$-pentanol

$$
\begin{aligned}
\hline M_{2}= & 88.15 \times 10^{-3} \mathrm{~kg} \mathrm{~mol}^{-1} \\
\sigma_{2}= & 6.667 \times 10^{-10} \mathrm{~m}, \varepsilon_{22} / k_{B}=304.1 \mathrm{~K} \\
c_{P 2}= & \left(3.8686+0.50451 * T-2.6394 \times 10^{-4}\right. \\
& \left.\times T^{2}+5.12 \times 10^{-8} \times T^{-3}\right) / M_{2} \mathrm{~J} \mathrm{~kg}^{-1} \mathrm{~K}^{-1} \\
\rho_{l}= & 270+10^{3}\left(1.930229 \times Z^{1 / 3}-8.414762 \times Z^{2 / 3}+19.226001 \times Z\right. \\
& \left.-18.559303 \times Z^{4 / 3}+6.555718 \times Z^{5 / 3}\right) \mathrm{J} \mathrm{mol}^{-1} \mathrm{~K}^{-1}, \\
& \text { where } Z=1-T / T_{c}, T_{c}=588.15 \mathrm{~K} \\
\gamma= & (26.85469-0.07889 \times(T-273.15)) \times 10^{-3} \mathrm{~N} \mathrm{~m}^{-1} \\
P_{\mathrm{eq}}= & 133.22 \times \exp (90.079043-9788.384 / T-9.9 \times \log T) \\
\lambda_{2}= & 1.88 \times 10^{-2}-9.068 \times 10^{-5} \times T+2.456 \times 10^{-1} \times T^{-2}
\end{aligned}
$$

Mixture

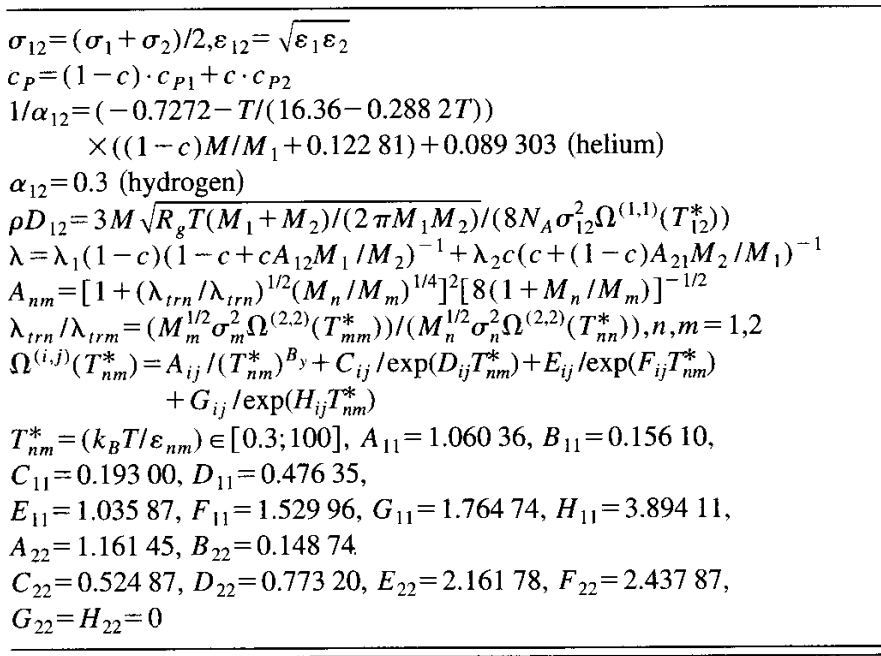

A solution of Eq. (35) may be obtained using the method of consequent approximations (the solution in the $N$ th approximation is designated by up-index ' $N$ ") and is given as

$$
\begin{aligned}
& c^{(N)}=1-\frac{1-c_{0}}{1+A^{(N)} F_{T}^{(N)}-G_{T}^{(N)}}, \\
& A^{(N)}=\left(\left(c_{1}-c_{0}\right) /\left(1-c_{1}\right)+G_{\tau}^{(N)}\right) / F_{\tau}^{(N)}, \quad N=1,2,3, \ldots, \\
& F_{\bar{T}}^{(N)}=\int_{1}^{\bar{T}} f^{(N)} d \bar{T}, F_{\tau}^{(N)}=\int_{1}^{\tau} f^{(N)} d \bar{T}, \\
& f^{(N)}=\frac{Q\left(c^{(N-1)}\right)}{1+\operatorname{Le}_{0}^{-1} A^{(N-1)} \cdot B\left(c^{(N-1)}, \bar{T}\right)},
\end{aligned}
$$

$G_{\bar{T}}^{(N)}=\int_{1}^{\bar{T}} g^{(N)} d \bar{T}, \quad G_{\tau}^{(N)}=\int_{1}^{\tau} g^{(N)} d \bar{T}$,

$g^{(N)}=\alpha_{12}^{(N-1)} \frac{c^{(N-1)}\left(1-c_{0}\right)}{\bar{T}\left(1-c^{(N-1)}\right)}$.

Equation (37) contains an unknown integration constant, $A^{(0)}$, and also functions, $F_{\bar{T}}^{(0)}, G_{\bar{T}}^{(0)}, F_{\tau}^{(0)}, G_{\tau}^{(0)}$, present in the zero approximation. Usually experiments in the static diffusion chamber are carried out using a light background gas (e.g., helium). When the molecular weight of the background gas is much smaller than the molecular weight of the vapor (e.g., $M_{1} \ll M_{2}$ ) and the vapor mass fraction is not close to unitity (e.g., approximately $c<1 / 2$ ) then the value of $Q(c)$ in Eq. (35) may be taken as $Q(c)=1$. Thus, using the approximation of a light background gas and neglecting thermal diffusion (e.g., $\alpha_{12}=0$ ), Eq. (33) for the boundary conditions given in Eqs. (20) and (21) assumes the simpler form,

$$
\frac{d}{d \vec{T}}\left(\frac{1-c_{0}}{1-c}\right)=\frac{A^{(0)}}{1+\operatorname{Le}_{0}^{-1} \bar{c}_{P 2}^{0} A^{(0)}(1-\bar{T})} .
$$

The solution to Eq. (38) can be written as

$$
\begin{aligned}
& c^{(0)}=1-\left(1-c_{0}\right)\left(1-\frac{\mathrm{Le}_{0}}{\bar{c}_{P 2}^{0}} \ln \left|1+A^{(0)}(1-\bar{T}) \frac{\bar{c}_{P 2}^{0}}{\mathrm{Le}_{0}}\right|\right)^{-1}, \\
& A^{(0)}=\frac{\mathrm{Le}_{0}}{\bar{c}_{P 2}^{0}(1-\tau)}\left(\exp \left(\frac{\bar{c}_{P 2}^{0}}{\mathrm{Le}_{0}} \frac{c_{0}-c_{1}}{1-c_{1}}\right)-1\right) .
\end{aligned}
$$

Thus, the system of simplified equations in (39) gives a solution to the transport in the SDC which does not account for thermal diffusion and applies for relatively small vapor mass fractions in a binary mixture. Equations (37) give the $N$-order iteration solution $(N=2,3,4, \ldots)$. This solution takes into account thermal diffusion and large vapor mass fractions. To evaluate any of these equations, appropriate values for the gas and vapor properties are needed, e.g., the molar weight $\left(M_{1}\right.$ and $\left.M_{2}\right)$, the force constants for the Lennard-Jones $(6-12)$ potential $\left(\sigma_{1}, \varepsilon_{1}\right.$ and $\left.\sigma_{2}, \varepsilon_{2}\right)$, the thermal diffusion factor $\left(\alpha_{12}\right)$, and the specific (or molar) heat capacity at constant pressure $\left(c_{P 1}\right.$ and $\left.c_{P 2}\right)$.

\section{RESULTS}

In order to investigate the difference between the two approaches for describing mass and energy transport in the SDC, namely, assuming a zero or a nonzero mass average velocity at the system boundaries we calculate values of vapor supersaturations based on the experimental $n$-pentanol nucleation results reported by Rudek $e t$ al. ${ }^{4}$ The vapor supersaturations correspond to the maximum of the theoretical nucleation rate as calculated using the classical (BDZ) nucleation theory,

$$
\begin{aligned}
J_{\text {theor }}= & \frac{V_{l}}{\left(k_{B} T\right)^{2}} \sqrt{\frac{2 \gamma}{\pi \cdot m_{2}}} S^{2} P_{\mathrm{eq}}^{2}(T) \\
& \cdot \exp \left(-16 \pi \cdot \gamma^{3} \cdot V_{l}^{2} / 3\left(k_{B} T\right)^{3} \cdot(\ln S)^{2}\right),
\end{aligned}
$$




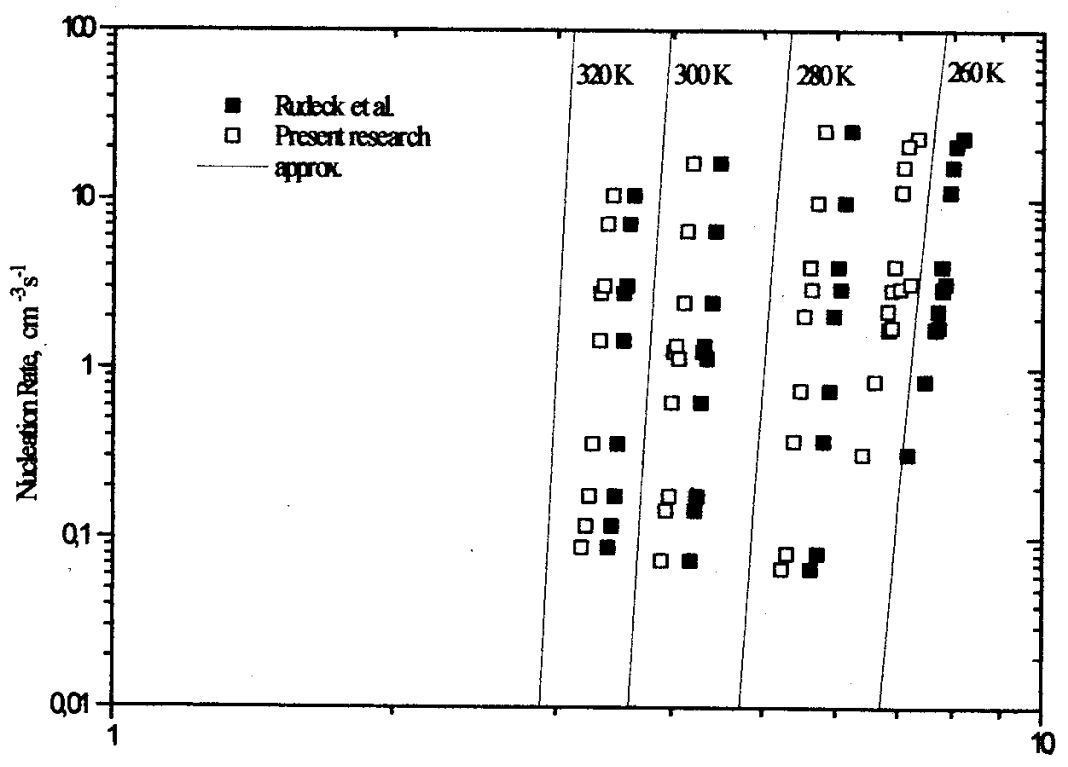

FIG. 1. Nucleation rates, $J$, vapor supersaturation, $S$, of $n$-pentanol in helium [Rudek (Ref. 4)] and the same data analyzed using the present research assumptions. Solid lines are predictions calculated using Eq. (41) with a Tolman factor of $0.4 \times 10^{-10} \mathrm{~m}$ for surface tension.

where $\gamma$ and $V_{l}$ are the surface tension and volume per molecule in the liquid phase, respectively; $S$ and $P_{\text {eq }}(T)$ are the vapor supersaturation ratio (or vapor activity) and equilibrium pressure, respectively; $T$ is the nucleation temperature; and $k_{B}$ is the Boltzmann constant. The thermophysical constants for $n$-pentanol and helium and their mixtures used in our analysis are, essentially, the same as used by Rudek et $a .^{4}$ and are shown in Table II.

The results of our calculations are shown in Fig. 1. In this figure we plot the measured nucleation rate as a function of the calculated supersaturation at four different nucleation temperatures. The solid symbols represent data as reported by Rudek $e t$ al. and the open symbols are the corresponding data analyzed using the method we describe above. As seen in the figure there is a significant difference in the calculated supersaturation for all of the data. The magnitude of this difference is quite large even for the relatively small vapor mass fractions at a nucleation temperature of $260 \mathrm{~K}$. We also note that the calculated nucleation temperatures from our analysis are slightly larger than those reported in Rudek et $a l .{ }^{4}$ We performed our calculations with and without the thermal diffusion term. We observed that the effect of thermal diffusion on the transport process is small and is not particularly essential to include in this comparison we are making of the effects of the different flux boundary conditions.

In order to compare the results of our analysis with $n$-pentanol nucleation rate data obtained earlier using a flow diffusion chamber, ${ }^{5}$ we utilized an expression for the nucleation rate fitted to the measured nucleation rate data that accounted for both temperature and total pressure. ${ }^{5}$ In that expression the pressure effect is taken in to account as

$$
\begin{aligned}
J_{\text {theor }}= & \frac{V}{(k T)^{2}} \sqrt{\frac{2 \sigma}{\pi \cdot m}} \cdot P^{2} \cdot \exp \left(-\frac{16 \pi \cdot \sigma^{2} \cdot V^{2}}{3(k T)^{3} \cdot(\ln S)^{2}}\right. \\
& \left.\cdot\left(1-\frac{2 \beta}{\ln S} \cdot \frac{P_{\mathrm{tot}}-P_{\mathrm{atm}}}{P_{\mathrm{atm}}}\right)\right),
\end{aligned}
$$

where $P_{\text {tot }}$ is total pressure and the fitting parameter, $\beta$, is expressed as $\beta=1.486 \cdot T_{r}^{3}$, where $T_{r}=T / T_{c}$ is the reduced temperature; $T_{c}$ is critical temperature of $n$-pentanol and equals $588.15 \mathrm{~K}$.

The fitting equation is based on the classical nucleation Eq. (40) with a Tolman factor for the size dependence of the surface tension as $\gamma=\gamma_{0}-\delta R_{g} T \rho_{l} \ln S / M_{2}$. The fitting parameter, $\delta$, equals to $0.4 \AA$ in this case. These calculations are shown in Fig. 1 as solid lines.

\section{CONCLUSIONS}

To describe the heat and mass transfer in the SDC, we apply the Navier-Stokes equations for a system with the axial symmetry and stationary vapor flow in the chamber axial direction. The heat and mass transfer equations in vector form are written and solved for the vapor flow boundary conditions. From our results one can see that there is a significant difference in the calculated vapor supersaturation for the SDC data. The magnitude of this difference is quite large even for the relatively small vapor mass fractions. We also note that the calculated nucleation temperatures from our analysis are slightly larger than usually reported. We performed our calculations with and without the thermal diffusion term. We observed that the effect of thermal diffusion on the transport process is relatively small and is not particularly essential to include in this comparison we are making of the effects of the different flux boundary conditions

\section{ACKNOWLEDGMENT}

The work at Clarkson University was supported by the National Science Foundation under Grant No. CTS 99776615 .

\section{APPENDIX}

The full system of mass and heat transport equations vector form (see Refs. 9 and 10) is given as 
(1) Mass balance for a nonreaction binary system (continuity equation),

$\nabla \cdot(\rho \mathbf{v})=0$,

where $\rho$ is the density of the mix, and $\mathbf{v}$ is a vector of mass average (hydrodynamic) velocity.

(2) Mass balance (continuity equation) for a vapor (2nd component),

$\rho \mathbf{v} \cdot \boldsymbol{\nabla} c_{2}+\operatorname{div} \mathbf{J}_{2}=0$.

Here, $c_{2}=m_{2} n_{2} / \rho$ is the mass concentration of the second component $\left(c_{1}+c_{2}=1\right) ; \mathbf{J}_{2}=m_{2} n_{2} \mathbf{V}_{2}$ is the vector for the mass diffusion flux of the second component $\left(\mathbf{J}_{1}+\mathbf{J}_{2}=0\right)$; and $\nabla$ is the differential operator. Here $m_{2}, n_{2}$, and $\mathbf{V}_{2}$ are molecule mass, number of molecules per unit of volume, and diffusion velocity of second component, respectively. ${ }^{12}$

(3) Equation of motion,

$\rho(\mathbf{v} \cdot \nabla) \mathbf{v}=2 \operatorname{Div}(\mu \mathbf{S})-\nabla(p+(2 / 3) \mu \operatorname{div}(\mathbf{v}))+\rho \mathbf{g}$,

where $\mathbf{g}$ is the acceleration of gravity, $p$ is pressure, $\mu$ is the dynamic viscosity, and $\mathbf{S}$ is the deformation rate tensor.

(4) Energy transport equation,

$$
\rho(\mathbf{v} \cdot \boldsymbol{\nabla} h)=\mathbf{v} \cdot \boldsymbol{\nabla} p+2 \mu(\mathbf{S})^{2}-(2 / 3) \mu\left(\operatorname{div}^{2} \mathbf{v}\right)-\operatorname{div}(\mathbf{q}),
$$

where $h$ is the specific enthalpy of the binary mix, and $\mathbf{q}$ is the energy flux vector (heat flux). The diffusion mass flux for eachcomponent of the binary system is expressed as

$$
\begin{aligned}
& \mathbf{J}_{1}=-\mathbf{J}_{2}=m_{1} m_{2} D_{12}\left(d_{2}-k_{T 1} \nabla \ln T\right) n^{2} / \rho, \\
& \mathbf{d}_{2}=-\nabla\left(n_{2} / n\right)+\left(n_{2} / n-n_{2} m_{2} / \rho\right) \nabla \ln (p),
\end{aligned}
$$

where $D_{12}$ is binary diffusivity, $k_{T 1}$ is the thermo-diffusion relation, defined through the integrals, $\Omega^{(1, S) *}$ and calculated on the basis of the Lennard-Jones potential. It is defined thus that the first-component transfers from the hot region to the cold for $k_{T 1}>0$ and transfers from the cold region to the hot for $k_{T 1}<0\left(k_{T 1}=-k_{T 2}\right)$. Considering that $c_{1}+c_{2}=1$, expression (A5) can be written as

$$
\begin{aligned}
\mathbf{d}_{2}= & c_{2}\left(M^{2} / M_{1} M_{2}\right)\left(\nabla \ln \left(c_{2}\right)+\left(1-c_{2}\right)\left(M_{1}-M_{2}\right) / M\right. \\
& \cdot \nabla \ln (p)), \\
\mathbf{J}_{1}= & -\mathbf{J}_{2}=c_{2} \cdot \rho \cdot D_{12}\left(\nabla \ln \left(c_{2}\right)\right. \\
& \left.+\left(1-c_{2}\right) \frac{M_{1}-M_{2}}{M} \nabla \ln (p)+\frac{k_{T 2}}{c_{2}} \frac{M_{1} M_{2}}{M^{2}} \nabla \ln (T)\right) .
\end{aligned}
$$

The heat flux can be expressed in the first approximation as

$$
\mathbf{q}=-\lambda \nabla T+\sum_{i=1}^{2} h_{i} \mathbf{J}_{i}+\frac{k T}{n} \sum_{i, j=1}^{2} \frac{n_{j} D_{i}^{T}}{m_{i} D_{i j}}\left(\mathbf{V}_{i}-\mathbf{V}_{j}\right) .
$$

Representing the diffusion velocity using mass flux expressions, the heat flux may be rewritten as

$$
\mathbf{q}=-\lambda \nabla T+\left(h_{2}-h_{1}+\frac{k_{T 2}}{c_{2}\left(1-c_{2}\right)} \frac{R T}{M}\right) \mathbf{J} .
$$

In a cylindrically symmetrically $(\partial / \partial \varphi=0)$ system, $\left(x_{1}, x_{2}, x_{3}\right)=(r, \varphi, z)$, divergences of the deformation rate tensor and the velocity are presented by Eqs. (A8) and (A9), respectively,

$$
\begin{aligned}
& (\operatorname{Div}(\mathbf{S}))_{r}=\frac{1}{r} \frac{\partial}{\partial r}\left(r S_{r r}\right)+\frac{\partial}{\partial z}\left(S_{z r}\right), \\
& (\operatorname{Div}(\mathbf{S}))_{z}=\frac{1}{r} \frac{\partial}{\partial r}\left(r S_{r z}\right)+\frac{\partial}{\partial z}\left(S_{z z}\right), \\
& S_{r r}=\frac{\partial v}{\partial r}, \quad S_{z r}=S_{r z}=\frac{1}{2}\left(\frac{\partial v_{z}}{\partial r}+\frac{\partial v_{r}}{\partial z}\right), \\
& S_{z z}=\frac{\partial v_{z}}{\partial z}\left(\mathbf{S}^{2}=\left(S_{r r}\right)^{2}+2\left(S_{r z}\right)^{2}+\left(S_{z z}\right)^{2}\right), \\
& \operatorname{div}(\mathbf{v})=\frac{1}{r} \frac{\partial}{\partial r}(r v)+\frac{\partial}{\partial z}(u), \quad\left(u=v_{z}, v=v_{r}\right) .
\end{aligned}
$$

${ }^{1} \mathrm{~J}$. Smolik and P. E. Wagner, Nucleation and Atmospheric Aerosols 1996, edited by M. Kulmala and P. E. Wagner (Pergamon, Helsinki, Finland, 1996), p. 58.

${ }^{2}$ H. Lihavainen and Y. Viisanen, J. Aerosol Sci. 28, Suppl. 1, 23 (1998).

${ }^{3}$ C. C. M. Luijten, O. D. E. Baas, and M. E. H. van Dongen, J. Chem. Phys. 106, 4152 (1997).

${ }^{4}$ M. Rudek, J. L. Katz, I. Y. Vidensky, V. Zdimal, and J. Smolik, J. Chem. Phys. 111, 3623 (1999).

${ }^{5}$ M. P. Anisimov, P. K. Hopke, S. D. Shandakov, and I. I. Shvets, J. Chem. Phys. 113, 1971 (2000).

${ }^{6}$ (a) A. Bertelsmann and R. H. Heist, J. Chem. Phys. 106, 610 (1999); (b) 106, 624 (1997).

${ }^{7}$ F. T. Ferguson and J. A. Nuth, J. Phys. Chem. 111, 8013 (1999).

${ }^{8}$ F. Stratmann, V. Zdimal, M. Wilck, and J. Smolik, J. Aerosol Sci. 30, S75 (1999).

${ }^{9}$ J. O. Hirschfelder, C. F. Curtiss, and R. B. Bird, Molecular Theory of Gases and Liquids (Wiley, New York, 1954).

${ }^{10}$ L. G. Loycansky, Mechanics of Liquids and Gases (Nauka, Moscow, 1987), p. 840 (in Russian).

${ }^{11}$ R. C. Reid, J. M. Prausnitz, and T. K. Sherwood, The Properties of Gases and Liquids, 3rd ed. (McGraw-Hill, New York, 1977), p. 508.

${ }^{12}$ A. Bertelsmann and R. H. Heist, Aerosol. Sci. Technol. 28, 259 (1998). 